\section{Reader panel profile}

The BDJ appointed a Reader Panel in 2018; the 13 members are called upon by the editorial team for feedback, ideas and suggestions for the $B D J$.

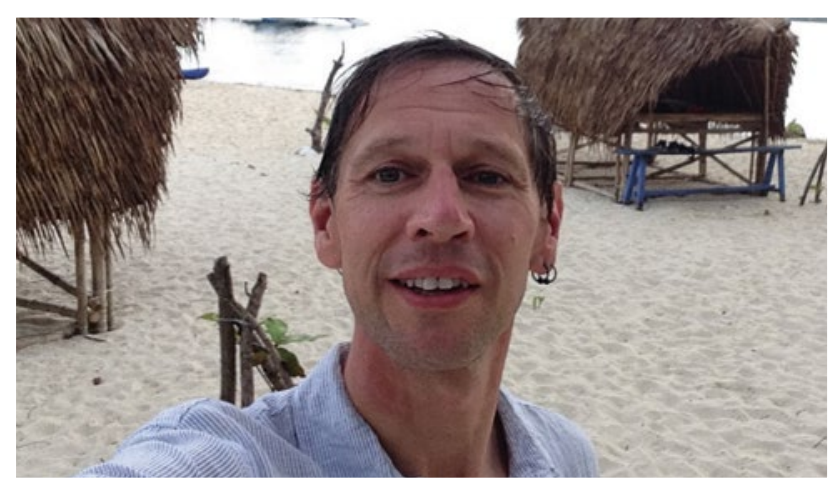

\section{Simon Threadgill}

Simon, from Newcastle, qualified in 2013 and is a GDP at Framwellgate Dental Practice, an NHS practice with five associates, five foundation dentists and a private dental hygienist.

Why did you become a dentist? I used to be a secondary school science teacher and I wanted a change of career. I have a decent science background and knew that the soft skills I had developed as a teacher would be very useful and well translatable to dentistry. I have also always liked helping people. Lastly, dentistry is a less demanding job than teaching and better remunerated.

Why did you join the BDJ Reader Panel? I have been a member of the BDA since the first year of dental school and I have always valued it as an organisation. It is nice to be in a more active role within it and to help, even in a small way, to influence the direction of articles in the $B D J$.

What recent $B D J$ paper would you recommend to our readers and why? The article in the 13 September 2019 issue CariesCare practice guide: consensus on evidence into practice. ${ }^{1}$ This is a fantastic update on caries diagnosis and management based on the latest in preventive and mimimally invasive caries protocols. A must-read.

\section{Have you ever changed your practice because of an article} you read in the BDJ? Yes. I had been to a number of courses and attended webinars and lectures on the new periodontal disease classification. I read the article in the January 2019 issue on the same only last month and immediately went into work the next day and began, for the first time, to grade and stage my periodontitis patients. $^{2}$

\section{References}

1. Martignon S, Pitts N B, Goffin G et al. CariesCare practice guide: consensus on evidence into practice. Br Dent J 2019; 227: 353-362.

2. Dietrich $\mathrm{T}$, Ower $\mathrm{P}$, Tank M et al. Periodontal diagnosis in the context of the 2017 classification system of periodontal diseases and conditions - implementation in clinical practice. Br Dent J 2019; 226: 16-22.

\section{Luncheon celebrates DCPs involved in research}

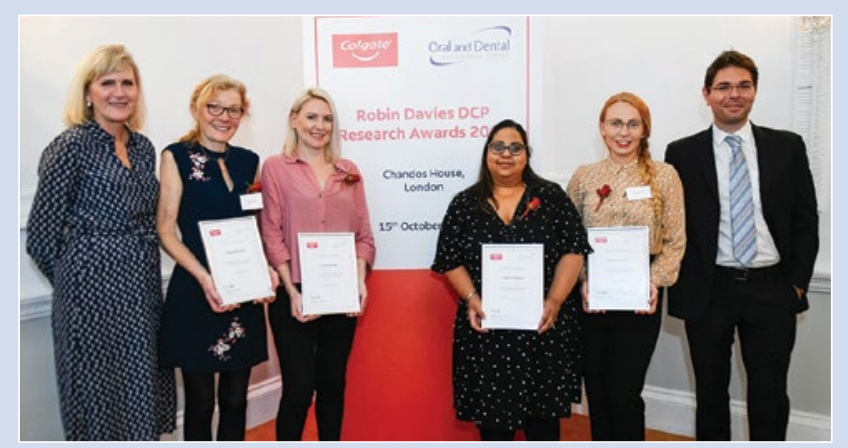

Awardees with the Colgate team

A celebratory luncheon for the 2019 recipients of the Robin Davies DCP Awards took place at Chandos House, London, in October, attended by leaders in dentistry from across the UK.

These awards, unique to dental care professionals (DCPs), are offered via a partnership between the Oral and Dental Research Trust (ODRT) and Colgate. They are offered for research conducted by DCPs to support investigations of clinical relevance, particularly in the field of preventive care.

The Keynote speaker, Donna Holden MBE, Postgraduate Dental Dean - Health Education England Northwest, said 'As a DCP, a former recipient of this award and as the first non-dentist Postgraduate Dental Dean I was delighted for the invitation to speak and proud to be associated with these awards which helped with my own career development'. Donna talked about the evolving role of DCPs, shared her vision for the workforce of the future and invited DCPs to explore research opportunities.

Professor Nairn Wilson, ODRT Trustee invited the 2019 awardees to outline their winning proposals.

Dental therapist Laura Brady is investigating the acceptability and implementation of digital photography to inform and motivate patients in NHS dental practice. Dental nurse Louise Belfield is investigating periodontal disease and angiogenesis: identifying a modifiable risk factor for oral cancer. Dental hygienist and dental therapist Janani Sivabalan is investigating a pilot project to assess the acceptability and effectiveness of a dental walk-in clinic for homeless people at the University of Portsmouth Dental Academy. Dental nurse Samantha Glover is investigating the identification of barriers to dental care for adults living with dementia in residential care.

Professor Wilson said: 'All awardees have shown great professionalism and justifiable success with their fantastic award submissions. The Robin Davies awards are unique globally in providing research support for DCPs and this luncheon is a wonderful opportunity to recognise research designed and delivered by DCPs. As a trustee of the ODRT it is an honour and a privilege to come together with Drs Lone Lenes and Emanuele Cotroneo from Colgate Scientific Affairs to celebrate the success of the 2019 awardees'.

Visit www.odrt.org.uk/colgate-dcp-awards/ to apply for the Robin Davies DCP Awards 2020; entries close on 7 February 2020. 